Research Paper

\title{
High GCLC level in tumor tissues is associated with poor prognosis of hepatocellular carcinoma after curative resection
}

\author{
Jialei Sun ${ }^{1,2,}{ }^{*}$, Chenhao Zhou $2,3,{ }^{*}$, Qianni Ma1,2, ${ }^{*}$, Wanyong Chen ${ }^{3,4}$, Manar Atyah ${ }^{3}$, Yirui Yin 3 , Peiyao Fu ${ }^{3}$, \\ Shuang $\mathrm{Liu}^{3}$, Bo Hu${ }^{3}$, Ning Ren ${ }^{3,4,}$, , Haijun $\mathrm{Zhou}^{1,2,}$ \\ 1. Liver Cancer Institute \& Zhongshan Hospital, Fudan University, Shanghai 200032, China \\ 2. Key Laboratory of Carcinogenesis \& Cancer Invasion, Ministry of Education, China \\ 3. Department of Liver Surgery, Zhongshan Hospital, Fudan University, Shanghai 200032, China \\ 4. Department of Surgery, Institute of Fudan-Minhang Acadamic Health System, Minhang Branch, Zhongshan Hospital, Fudan University, Shanghai, China \\ ${ }^{*}$ Equally contributed authors. \\ $\square$ Corresponding authors: Haijun Zhou, Liver Cancer Institute \& Zhongshan Hospital, Fudan University, Shanghai 200032, China; Key Laboratory of \\ Carcinogenesis \& Cancer Invasion, Ministry of Education, China. Tel: +86-21-64041990, Email: zhou.haijun@zs-hospital.sh.cn. Ning Ren, Department of Liver \\ Surgery, Zhongshan Hospital, Fudan University, Shanghai 200032, China; Department of Surgery, Institute of Fudan-Minhang Acadamic Health System, \\ Minhang Branch, Zhongshan Hospital, Fudan University, Shanghai, China. Tel: +86-21-64041990, Email: ren.ning@zs-hospital.sh.cn.
}

(c) Ivyspring International Publisher. This is an open access article distributed under the terms of the Creative Commons Attribution (CC BY-NC) license (https://creativecommons.org/licenses/by-nc/4.0/). See http://ivyspring.com/terms for full terms and conditions.

Received: 2018.09.06; Accepted: 2019.05.05; Published: 2019.06.02

\begin{abstract}
Glutamate-cysteine ligase catalytic subunit (GCLC) has been reported to overexpress in a variety types of cancer and be related with tumor progression and drug resistance. However, little has been known about GCLC's prognostic significance and biological roles in hepatocellular carcinoma (HCC). In the present study, we evaluated GCLC expression level using immunohistochemical staining (IHC) in tissue microarray (TMA) containing paired tumor and peritumoral liver tissues from 168 patients with HCC who received curative resection. GCLC levels in tumor tissues were significantly higher than in peritumoral liver tissues, and tumor GCLC level was associated with overall survival (OS) and disease-free survival (DFS). Five-year OS and DFS rates were $41.15 \%$ and $25.88 \%$ for the group with high tumor GCLC level, compared with $68.09 \%$ and $47.51 \%$ for the group with low tumor GCLC level $(P<0.001$ and $P=0.001$, respectively). Moreover, quantitative reverse transcription PCR ( $q R T-P C R$ ) analysis demonstrated that GCLC was transcriptionally activated in HCC tissues when comparing with peritumoral tissues. Tumor GCLC level, which correlated to tumor differentiation, microvascular invasion and BCLC stage, was independent prognostic factors for both OS $(P=0.006)$ and $D F S(P=0.003)$. Importantly, tumor GCLC level was still significantly associated with OS and DFS in patients with early HCC. GCLC-based nomogram models were further established and exhibit significantly higher predictive accuracy as compared with routine clinical staging systems. In conclusion, tumor GCLC is a potential prognostic biomarker for HCC patients after receiving curative resection.
\end{abstract}

Key words: GCLC; Hepatocellular carcinoma; Prognosis; Survival analysis; Nomogram

\section{Introduction}

Hepatocellular carcinoma (HCC) is an extremely malignant tumor with a high mortality rate worldwide. The general prognosis of this disease remains extremely poor despite improved clinical diagnosis and treatment strategies that have emerged during the past few decades [1]. Curative resection is the best method to provide long-term survival for patients with HCC, however, the high frequency of metastatic recurrence after curative treatments remains a major obstacle [2]. Identification of molecular markers is helpful for predicting clinical outcome in HCC patients and improving the selection of patients for adjuvant therapies after resection. But so far, there have not been satisfactory available 
biomarkers. Although several staging systems have been used to classify HCC, they have limitations for determining clinical outcome, especially in patients with early-stage disease who do not have obvious vascular invasion or regional lymph node or distant metastasis [3-4]. Therefore, it is urgently required to identify new biomarkers for predicting clinical outcome of patients after resection, particularly for patients with early stage disease.

Accumulating data suggests that oxidative stress, which is caused by high levels of reactive oxygen species (ROS) or impaired antioxidant defense systems, may play fundamental roles in cancer formation and progression [5]. Efforts have been made to kill cancer cells by targeting ROS-mediated mechanisms, but the results were unsatisfying due to constitutive overexpression of antioxidant genes in malignant cells [6]. Glutathione (GSH) is a critical antioxidant involving in both physiological and pathological processes. It has been revealed that increased GSH level in cancer cells contributes to cell proliferation, cell cycle progression, invasion and drug resistance [7-10]. Since GSH biosynthesis is a multi-step process that involves a series of synthetases, research focusing on GSH-related synthetases may help to discover novel diagnostic, prognostic biomarkers and potential therapeutic targets for cancer.

$\gamma$-glutamylcysteine synthetase (GCL) activity is closely related to the elevated GSH levels in many types of cancer [11]. As a member of GCL, GCLC (Glutamate-cysteine ligase catalytic subunit) is a rate-limited enzyme that involves in the first step of GSH biosynthesis. It has been reported that GCLC was aberrantly expressed in tumor tissues [12-14]. GCLC has been found to overexpress in liver metastases of colorectal cancer and promotes cancer cell survival [15]. Recently, some studies also reveal that GCLC activation are associated with anti-tumor drug resistance in breast, lung, liver, head and neck cancer [16-20]. All these evidences suggest that GCLC may act as an oncogene. However, GCLC has been rarely studied in HCC, so little is known about its prognostic significance and biological roles in HCC.

In the present study, we attempt to investigate the prognostic values of GCLC in HCC patients who had received curative resection. The matched HCC and peritumoral tissue samples from 168 patients with HCC were used to examine the expression levels of GCLC by immunohistochemical staining (IHC). The association of tumor GCLC level with overall survival (OS) and disease-free survival (DFS) as well as clinicopathological characteristics of patients was then analyzed. The mRNA expression level of GCLC was investigated in 29 pairs of HCC and peritumoral tissue samples using Quantitative reverse transcription PCR (qRT-PCR). The GCLC protein expression level and subcellular distribution also were analyzed in HCC cell lines and nontransformed hepatic cell line using Western blot (WB) and immunofluorescence (IF). Finally, prognostic nomograms by integrating tumor GCLC level and other independent prognostic factors were proposed to give accurate prediction of prognosis (OS and DFS).

\section{Material and Methods}

\subsection{Patient samples and follow-up}

168 HCC patients who had received curative resection from January 2009 to January 2010 in Zhongshan Hospital, Fudan University, were enrolled at random and followed up strictly. The criteria of patient inclusion and exclusion were as following: (1) no systemic or local treatments were received before hepatectomy, (2) no cases with extrahepatic metastasis before hepatectomy, (3) the postoperative pathologic diagnosis of all the patients enrolled was HCC, (4) all patients underwent curative resection, (5) no infectious evidence or other inflammatory conditions except for viral hepatitis, (6) all patients had complete clinicopathologic information. Clinical samples were collected from patients after obtaining informed consent in accordance with a protocol approved by the Ethics Committee of Zhongshan Hospital, Fudan University.

The clinicopathological data retrospectively reviewed included age, gender, alanine aminotransferase (ALT), aspartate aminotransferase (AST), alpha-fetoprotein (AFP), $\gamma$-glutamyltransferase ( $\gamma$-GT), carcino-embryonic antigen (CEA), carbohydrate antigen 19-9 (CA19-9), presence of hepatitis B virus (HBV) surface antigen ( $\mathrm{HbsAg}$ ), ascites, liver cirrhosis, microvascular invasion and tumor number, size, encapsulation, differentiation. All the laboratory examinations were conducted within 5 days before surgery. The clinical stages of the patients were evaluated by Tumor Node Metastasis (TNM) staging system of AJCC $7^{\text {th }}$ edition and Barcelona Clinic Liver Cancer (BCLC) staging system.

All patients were followed up to 30 June 2016, with a median follow-up of 50.5 months (range from 0.2 to 87.5 months). The follow-up included abdominal ultrasound, chest imaging examination and serum alpha-fetoprotein (AFP) with intervals of three months or less during the first year, three to six months during the following two years, and one a year afterwards. CT scanning or magnetic resonance imaging (MRI) was done when recurrence was suspected. A diagnosis of recurrence was based on typical appearance on CT scans and/or MRI. 67.86\% 
$(114 / 168)$ of all enrolled patients developed HCC recurrence during the follow-up period. OS was defined as the time from the surgery to either death or the last follow-up (June 2016). DFS was defined as the interval between the dates of surgery and recurrence. For patients who had not been diagnosed with recurrence, DFS was censored on the date of death or the last follow-up.

\subsection{Cell lines and cell culture}

Human HCC cell lines MHCC97H, MHCCLM3 were obtained from Liver Cancer Institute, Zhongshan Hospital, Fudan University. SMMC-7721, PLC/PRF/5 and nontransformed hepatic cell line L02 were purchased from the cell bank of the Chinese Academy of Science (Shanghai, China). All the cells were cultured with Dulbecco's modified Eagle's medium (DMEM) (Hyclone, Logan, UT, USA) supplemented with $10 \%$ fetal bovine serum (Invitrogen, Carlsbad, CA, USA) and grew at $37^{\circ} \mathrm{C}$ and $5 \% \mathrm{CO}_{2}$ in the incubator.

\subsection{Immunohistochemistry analysis}

Tissue microarray (TMA) was constructed as described previously, and IHC analysis was performed as described previously [20]. Briefly, the prepared sections were incubated with GCLC antibody (1:100, sc-390811, Santa Cruz, CA, USA) at $4^{\circ} \mathrm{C}$ overnight. The horseradish peroxidase (HRP)-conjugated secondary antibody was incubated at $37^{\circ} \mathrm{C}$ for 45 minutes and then diaminobenzidine (DAB) solution (Dako REAL ${ }^{\mathrm{tm}}$ EnVision TM Detection System, Denmark. Cat No\#K5007) was applied. The nuclei were counterstained with Harris' Hematoxylin. PBS and monoclonal Mouse IgG (isotype control, 1:1000, Abacm, Cambridge, UK. Cat No\#172730) were set as negative control. Two pathologists who were blinded to the clinical data independently assessed the IHC staining of GCLC using a semiquantitative histological score (H-score) approach, which combines the intensity and number of cells positive for GCLC expression. Staining intensity of GCLC was categorized as follows: $0(-) ; 1(+) ; 2(++)$ and $3(+++)$. The mean percentage of positively stained cells was scored as follows: $0(<5 \%) ; 1(5-25 \%) ; 2(26-50 \%) ; 3$ $(51-75 \%)$ and $4(76-100 \%)$. The final scores were generated by multiplying the staining proportion scores with staining intensity scores. Based on the final scores, GCLC expression levels were classified into four grade: negative (0), or weak (1-4), or moderate (6-8), or strong (9-12).

\subsection{Immunoblotting}

For immunoblotting, cells were washed with cold PBS for three times and lysed in the RIPA extraction regent (Pierce Biotechnology, Rockford, IL,
USA) containing $1 \%$ protease inhibitor cocktail (Roche, France) and phosphatase inhibitor (Sigma, France) for 1 hour on ice. After clarified by centrifugation for $10 \mathrm{~min}$ at $12,000 \times \mathrm{g}$, the supernatant of cell lysates was added with 5X SDS-PAGE loading buffer (Beyotime, Shanghai, China. Cat No\#P0015) and then heated to $95-100^{\circ} \mathrm{C}$ for 10 minutes. $20 \mu \mathrm{g}$ total protein were loaded per well for blotting and electrotransfer to $0.45 \mu \mathrm{m}$ PVDF membrane (Immobion-P Transfer Membrane, Millipore, Billerica, MA, USA. Cat No\# IPVH 00010). Following 5\% non-fat dry milk blockage of membranes, specific primary antibody for GCLC (1:1000, sc-390811, Santa Cruz, CA, USA) and GAPDH (1:2000; Sigma-Aldrich, USA. Cat No\# G9295) serving as internal reference were incubated overnight in $4^{\circ} \mathrm{C}$. After washed three times for 5 minutes with TBST, the membranes were incubated with secondary antibody (Peroxidaseconjugated AffiniPure Goat anti-Mouse IgG $(\mathrm{H}+\mathrm{L})$, 1:2000; Jackson ImmunoResearch, USA. Cat No\#111-035-003) for 2 hours and then exposed to Tanon-5200 Chemiluminescent Imaging System (Tanon, China) for imaging. All the experiments were performed for three times.

\subsection{Quantitative reverse transcription PCR}

HCC tissue samples were lysed for acquirement of total RNA by TRIzol (Takara, Japan). PrimeScript RT reagent kit (Takara, Japan) was used for reverse-transcription. Approximately $1 \mu \mathrm{g}$ of total RNA were used for each PCR reaction, performed with SYBR ${ }^{\circledR}$ Premix ExTaq ${ }^{\mathrm{TM}}$ (Takara, Japan) on a ABI Prism 7500 Sequence Detection system (Applied Biosystems, Foster City, CA, USA). The sequences of primers were listed as following: GCLC-forward: 5'-TTAGGCTGTCCTGGGTTCAC-3'; GCLC-reverse: 5'-TCGCTCCTCCCGAGTTCTAT-3'; GAPDH-forward: 5'-CTGGGCTACACTGAGCACC-3'; GAPDHreverse: 5'-AAGTGGTCGTTGAGGGCAATG-3'. All the experiments were performed for three times.

\subsection{Immunofluorescence}

$1 \times 10^{5}$ cells were seeded on glass coverslips and grew for three days. Cells were washed three times with PBS and fixed in $4 \%$ paraformaldehyde, permeabilized in $0.25 \%$ Triton X-100 and then blocked with $5 \%$ goat serum. Sections were incubated with GCLC primary mouse antibody (1:100, sc-390811, Santa Cruz, CA, USA) overnight at $4^{\circ} \mathrm{C}$. After washed with PBST for three times, the coverslips were incubated with Alexa Fluor ${ }^{\circledR}$ 488-conjugated goat anti-mouse secondary antibody (1:100, Abcam, Cambridge, UK. Cat No\#ab150117) for 2 hours in dark. Neclei were counterstained with 2-(4-Amidinophenyl)-6-indolecarbamidine dihydrochloride (Beyotime, Shanghai, China. Cat No\#C1005). 
All the sections were observed under a fluorescence microscope (Olympus).

\subsection{Statistical analysis}

Statistical analyses were performed with SPSS software (23.0; IBM, Armonk, NY, USA). The $t$ test or Pearson's correlation test was used to compare quantitative variables and qualitative variables were analyzed by Pearson $\chi^{2}$ test or Fisher's exact test. Kaplan-Meier analysis was used to determine OS and DFS. Log-rank test was used to compare patients' survival between subgroups. Univariate Cox proportional hazards regression models were performed to identify relevant variables. For significant factors, a multivariate Cox regression analysis was applied in a stepwise manner. The data shown represent mean values of three independent experiments and are expressed as mean \pm standard deviation (SD). Statistical tests were all two-tailed and $P$ value less than 0.05 was considered statistically significant.

Leave-one-out cross-validation procedure ( $R$ project version 3.5.1 package) was performed to validate our main findings as previous report [21]. In brief, each time one sample was taken as a test sample, and all the remaining samples are used as training sets. This process was repeated until all the samples were traversed. $P$-values were derived from level-one-out cross-validation for OS and DFS, respectively.

Based on the results from multivariate analysis, nomogram models were established by $\mathrm{rms}$ in $\mathrm{R}$ project version 3.4 .2 package (http://www.rproject.org/). The comparison between C-indexs was then carried out using Hanley-McNeil test. Concordance index (C-index) was used to assess the predictive accuracy of the constructed nomogram. The larger the C-index, the more accurate the prognostic nomogram was.

\section{Results}

\subsection{GCLC levels in tissues from HCC patients and cell lines.}

Immunohistochemical staining for GCLC was performed on the paired HCC and peritumoral tissues from 168 patients with HCC who had received curative resection. GCLC staining was mainly on tumor cells in tumor tissues and hepatocytes in peritumoral tissues. GCLC levels were evaluated according to immunohistochemistry (IHC) scoring and classified into four grades: strong, moderate, weak and negative (Fig. 1A). We found that tumor tissues had significantly increased percentage of strong (25\%) and moderate (27.98\%) staining of GCLC as compared with peritumoral tissues (Strong, 8.93\%; moderate, 25.6\%, $P=0.010$ ) (Fig. 1B). To confirm whether GCLC was transcriptionally activated in HCC, the mRNA expression levels of GCLC were determined in 29 pairs of HCC and peritumoral tissues which were randomly selected from total 168 cases. As shown in Fig. 1C, mRNA expression levels in HCC tissues were also significantly elevated as compared with their paired peritumoral tissues $(P=0.002)$.

For HCC cell lines (MHCC97H, MHCCLM3, SMMC-7721, PLC/PRF/5), both mRNA and protein expression levels of GCLC were significantly increased as compared with the nontransformed hepatic cell line L02 (Fig. 1D and 1E). Next, to investigate subcellular distribution of GCLC in HCC and nontransformed hepatic cells, MHCC97H, MHCCLM3, SMMC-7721, PLC/PRF/5 and L02 cells were subjected to immunofluorescence staining of GCLC. It was observed that GCLC was mainly found in cytoplasm of all these cells (Fig. 1F). Consistent with our findings in TMA, staining intensity of GCLC in MHCC97H, MHCCLM3, SMMC-7721 and PLC/PRF/ 5 cells was obviously higher than that in L02 cells. Moreover, from the Oncomine Microarray Database, we found that GCLC was also overexpressed in other types of cancer such as breast, lung, prostate and renal cancers, which suggest that overexpression of GCLC in cancer is a common phenomenon (Fig. S1A).

\subsection{Correlation between tumor GCLC level and clinicopathologic characteristics}

Based on the grades of GCLC IHC staining, the 168 patients were divided into two subgroups: high tumor GCLC $(n=89)$ and low tumor GCLC $(n=79)$. The subgroup of high tumor GCLC was composed of the HCC patients with strong and moderate GCLC expression in their tumor tissues, while the subgroup of low tumor GCLC was made up of the HCC patients with GCLC negative and weak expression in their tumor tissues. After the definition of subgroups, the association of tumor GCLC level with clinicopathologic characteristics was analyzed. As shown in Table 1, GCLC level correlated positively with tumor differentiation $(P=0.004)$, microvascular invasion $(P=0.029)$ and BCLC stage $(P=0.020)$, but it was not related to any other clinicopathologic feature.

\subsection{Association of tumor GCLC level with prognosis of HCC patients.}

Kaplan-Meier analyses for OS and DFS were performed in the 168 patients using the above classification standards as the cut-off for the definition of the subgroups. As shown in Fig. $2 \mathrm{~A}$ and $2 \mathrm{~B}$, the 
patients in the high-GCLC subgroup had a significantly shorter OS and DFS than those in the low-GCLC subgroup. The OS probabilities at 1, 3 and 5 years in the high-GCLC subgroup were $76.41 \%$, $49.44 \%$ and $41.15 \%$, respectively, which were significantly lower than in the low-GCLC subgroup (88.61\%, $73.35 \%$ and $68.09 \%$, respectively; $P<0.001)$. The probabilities of DFS at 1,3 and 5 years of the high-GCLC subgroup (65.74\%, 33.45\% and $25.88 \%$, respectively) were also significantly lower than those

A.

HCC tissue

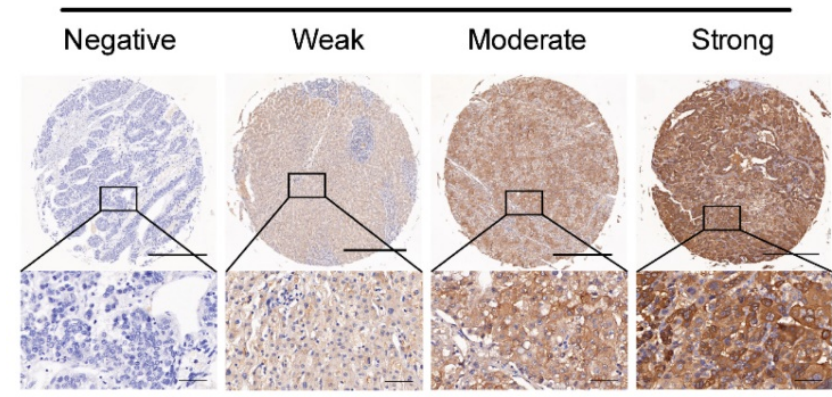

Peritumoral tissue
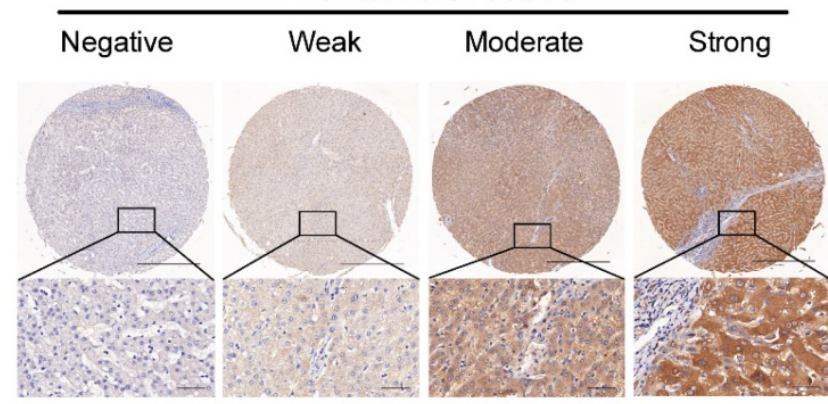

D.

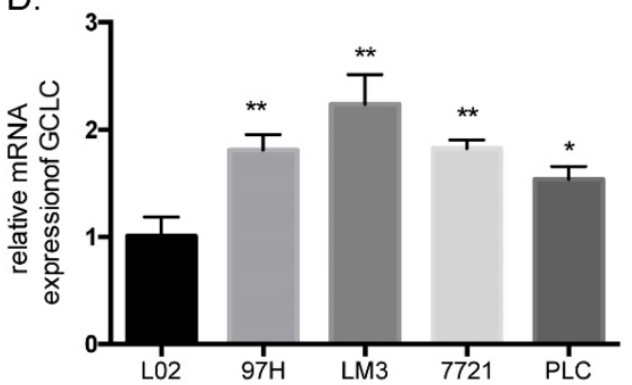

F.

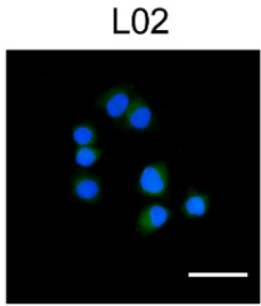

$\mathrm{MHCC97H}$
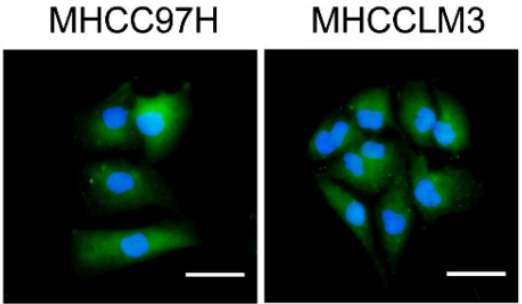

of the low-GCLC subgroup $(78.48 \%, 59.49 \%$, and $47.51 \%$, respectively; $P<0.001)$. To confirm prognostic significance of GCLC, we performed an internal validation procedure (leave-one-out cross-validation) on the entire patient cohort used in the present study. As shown in Table S1, all $P$-values of OS and DFS derived from leave-one-out cross-validation were statistically significant $(P<0.05)$. These results further validated our findings.
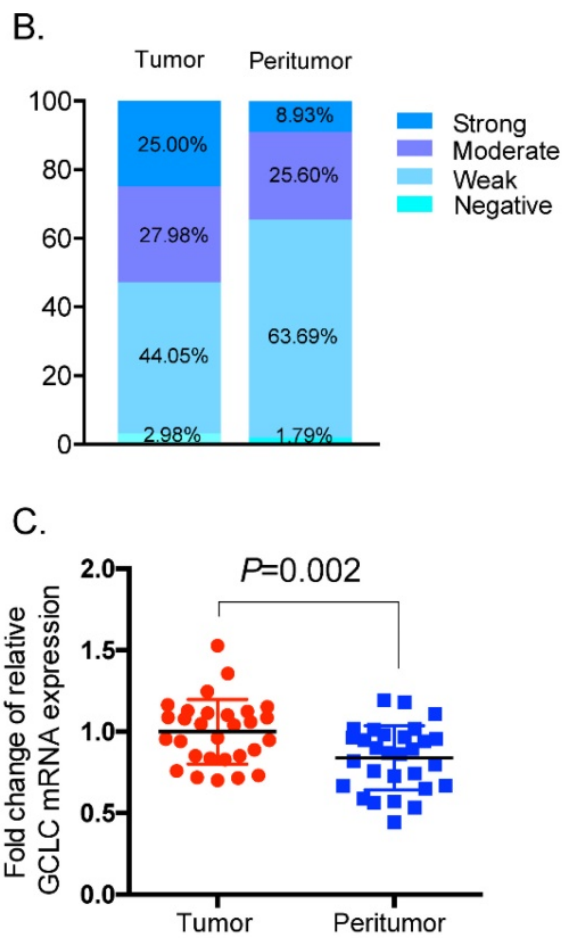

E.

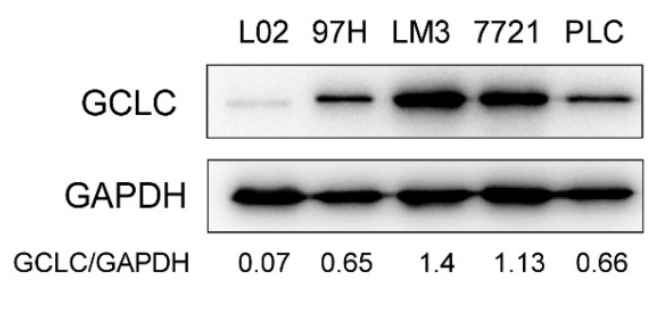

Figure 1. GCLC is overexpressed in HCC tissues as compared with peritumoral tissues. (A)Representative IHC staining of GCLC in HCC and peritumoral tissues,

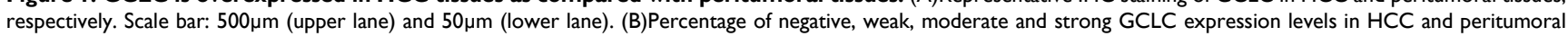
tissues. (C)The comparison between GCLC mRNA level in $\mathrm{HCC}$ and peritumoral tissues. GAPDH is set as internal control. $P<0.05$ is considered as statistically significant, paired Student $t$ test. (D, E) qRT-PCR and immunoblotting analysis of GCLC expression in HCC cell lines and nontransformed hepatic cell line. (F)Representative immunofluorescence images of GCLC in HCC cell lines and nontransformed hepatic cell line. Green fluorescence: GCLC, blue fluorescence: nucleus. Scale bar: $50 \mu m$. 
A.

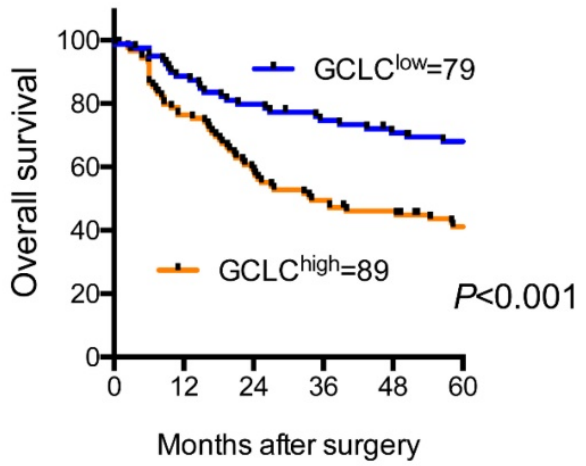

C.

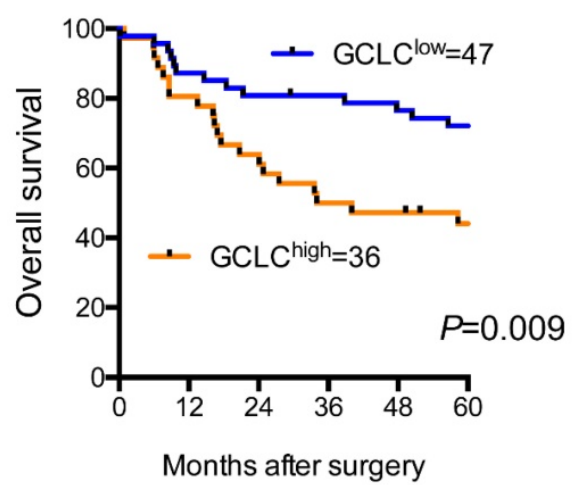

E.

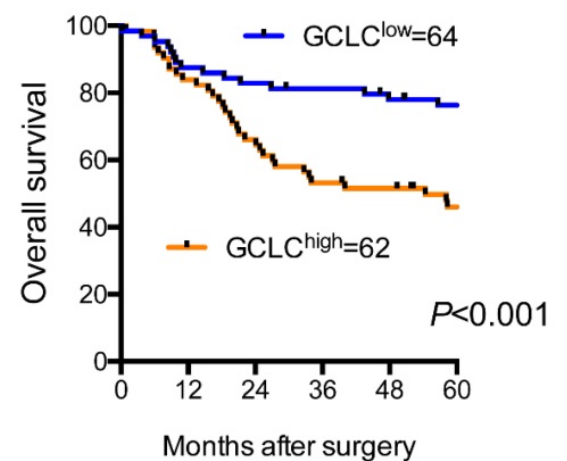

B.

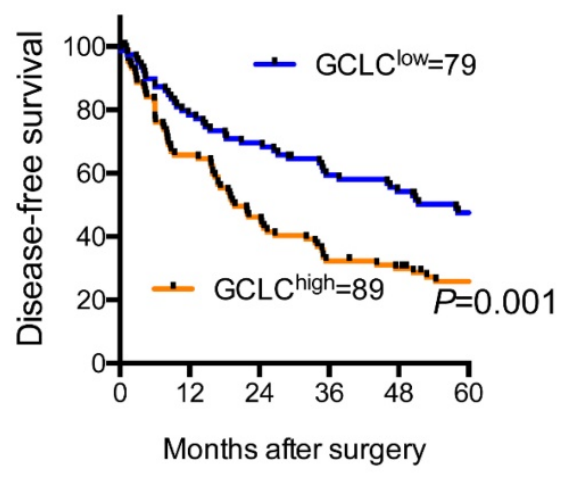

D.

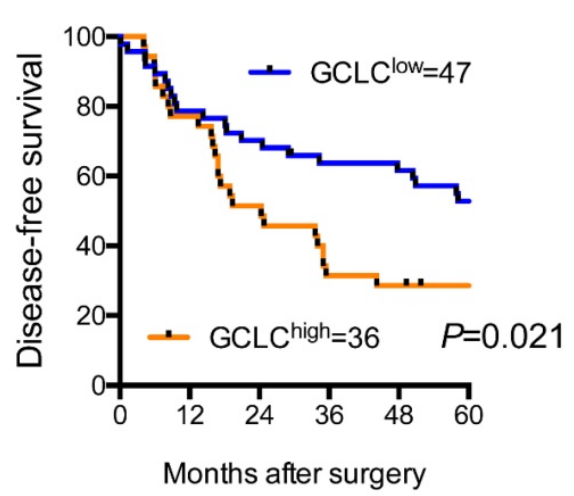

F.

TNM I-II

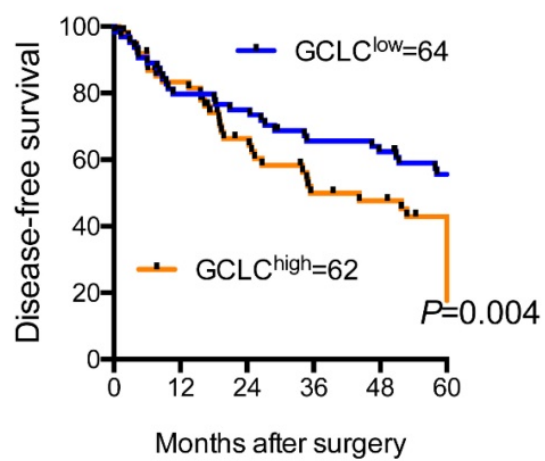

Figure 2. High tumor GCLC level predicts poor clinical outcome in HCC patients. Kaplan-Meier curves for overall survival (OS) and disease-free survival (DFS) in (A, B) all cases ( $n=168),(C, D) B C L C$ stage 0 -A subgroup $(n=83),(E, F)$ TNM stage I-ll subgroup $(n=126)$. OS and DFS are analyzed by Kaplan-Meier method using the log-rank test. $P<0.05$ is considered as statistically significant. BCLC, Barcelona Clinic Liver Cancer staging system. TNM, Tumor Node Metastasis staging system of AJCC 7th edition.

We further evaluated the prognostic value of tumor GCLC for patients with early-stage HCC. BCLC staging system and TNM staging system were used to determine HCC stage of patients. BCLC stage 0 -A and TNM stage I-II were defined as early stage of HCC. For the patients with HCC of BCLC stage 0-A (Fig. 2C and 2D), our data revealed that the probabilities of OS at 3 and 5 years in the high-GCLC subgroup $(50.00 \%$ and $44.07 \%$, respectively) were significantly lower than those in the low-GCLC subgroup $(80.85 \%$ and $72.11 \%$, respectively; $P=0.009$ ). The DFS probabilities at 3 and 5 years of the
high-GCLC subgroup were $40.31 \%$ and $28.57 \%$, respectively, which also were significantly lower than those of the low-GCLC subgroup $(74.90 \%$ and $52.77 \%$, respectively; $P=0.021)$. Similarly, for the patients with HCC of TNM stage I-II (Fig. 2E and 2F), high tumor GCLC level was significantly associated with shorter OS and DFS ( $\mathrm{P}<0.001$ and $\mathrm{P}=0.004$, respectively.).

\subsection{Univariate and multivariate analyses of the prognostic abilities of tumor GCLC level}

In Univariate analysis, serum AFP, CA19-9, AST, $\mathrm{Y}$-GT, tumor size, and tumor GCLC level were all 
significantly related to OS and DFS of HCC patients (Table 2). Besides, tumor differentiation and microvascular invasion were associated with OS while ALT and presence of ascites were associated with DFS. Age, gender, CEA, tumor number, serum HBsAg, liver cirrhosis, tumor encapsulation showed no prognostic significance for OS and DFS. The significant factors from univariate analysis were then adopted to multivariate Cox proportional hazards analysis. The prognostic values of tumor GCLC level for OS $(\mathrm{HR}=1.893, P=0.006)$ and DFS $(\mathrm{HR}=1.818$, $P=0.003$ ) were independent of the other clinical variables tested. In addition, serum AFP, $\gamma$-GT, tumor size also served as independent prognostic factors for OS and DFS. Furthermore, it was found that the tumor GCLC level was still significantly associated with 5-year OS and DFS in the patients with early stage disease (Fig. 3A and 3B).

\subsection{Prognostic value of GCLC-based nomograms for HCC}

We further established two new prognostic nomograms for OS and DFS respectively by combining tumor GCLC level and other independent prognostic factors identified by the multivariate analyses (Fig. 4A and 4B). To validate accuracy of the two prognostic nomograms, the calibration curves were drawn. As shown in Fig. 4C, 4D, 4E and 4F, there were high consistencies between nomogrampredicted values and the actual observed outcome. We next asked whether the GCLC-based nomograms could improve predictive accuracy for HCC. As listed in Table 3, GCLC-based nomogram for OS exhibited better predictive performance when compared with TNM staging system $(P<0.001)$ and BCLC staging system $(P<0.001)$ according to $C$-index (Harrell's concordance index). Likewise, the predictive accuracy of the GCLC-based nomogram for DFS was also higher than that of other routine staging systems.

Table 1. Correlation between tumor GCLC level and clinicopathologic characteristics in HCC patients.

\begin{tabular}{|c|c|c|c|c|c|}
\hline \multirow[t]{2}{*}{ Characteristics } & & \multirow{2}{*}{$\begin{array}{l}\text { Patients } \\
\text { Number (\%) } \\
\end{array}$} & \multicolumn{2}{|c|}{ Tumor GCLC level } & \multirow[t]{2}{*}{$P$ value } \\
\hline & & & Low $(n=79)$ & High $(n=89)$ & \\
\hline \multirow[t]{2}{*}{ Age, years } & $\leq 50$ & 73(43.45) & 33 & 40 & 0.756 \\
\hline & $>50$ & $95(56.55)$ & 46 & 49 & \\
\hline \multirow[t]{2}{*}{ Gender } & Female & $21(12.5)$ & 11 & 10 & 0.646 \\
\hline & Male & $147(87.5)$ & 68 & 79 & \\
\hline \multirow[t]{2}{*}{$\mathrm{HbsAg}$} & Negative & $27(16.07)$ & 12 & 15 & 0.835 \\
\hline & Positive & $141(83.93)$ & 67 & 74 & \\
\hline \multirow[t]{2}{*}{$\mathrm{AFP}, \mathrm{ng} / \mathrm{ml}$} & $\leq 20$ & $60(35.71)$ & 31 & 29 & 0.421 \\
\hline & $>20$ & $111(64.29)$ & 48 & 60 & \\
\hline \multirow[t]{2}{*}{$\mathrm{CEA}, \mathrm{ng} / \mathrm{ml}$} & $\leq 5$ & $155(92.26)$ & 75 & 80 & 0.259 \\
\hline & $>5$ & $13(7.74)$ & 4 & 9 & \\
\hline \multirow[t]{2}{*}{ CA19-9, U/ml } & $\leq 36$ & $130(77.38)$ & 61 & 69 & 1 \\
\hline & $>36$ & $38(22.62)$ & 18 & 20 & \\
\hline \multirow{2}{*}{$\mathrm{ALT}, \mathrm{U} / \mathrm{L}$} & $\leq 40$ & $98(58.33)$ & 46 & 52 & 1 \\
\hline & $>40$ & 70(41.67) & 33 & 37 & \\
\hline \multirow[t]{2}{*}{ AST, U/L } & $\leq 37$ & $118(70.24)$ & 56 & 62 & 1 \\
\hline & $>37$ & $50(29.76)$ & 23 & 27 & \\
\hline \multirow[t]{2}{*}{ Y-GT, U/L } & $\leq 54$ & 69(41.07) & 38 & 31 & 0.087 \\
\hline & $>54$ & $99(58.93)$ & 41 & 58 & \\
\hline \multirow[t]{2}{*}{ Ascites } & Absent & 159(94.64) & 75 & 84 & 1 \\
\hline & Present & $9(5.36)$ & 4 & 5 & \\
\hline \multirow{2}{*}{ Liver cirrhosis } & No & $28(16.67)$ & 13 & 15 & 1 \\
\hline & Yes & 140(83.33) & 66 & 74 & \\
\hline \multirow[t]{2}{*}{ Tumor number } & Single & $140(83.33)$ & 70 & 70 & 0.991 \\
\hline & Multiple & $28(16.67)$ & 9 & 19 & \\
\hline \multirow[t]{2}{*}{ Tumor size, $\mathrm{cm}$} & $\leq 5$ & $84(50)$ & 37 & 47 & 0.537 \\
\hline & $>5$ & $84(50)$ & 42 & 42 & \\
\hline \multirow[t]{2}{*}{ Tumor encapsulation } & Complete & 91(54.17) & 45 & 46 & 0.537 \\
\hline & None & $77(45.83)$ & 34 & 43 & \\
\hline \multirow[t]{2}{*}{ Tumor differentiation } & I-II & $104(61.9)$ & 58 & 46 & 0.004 \\
\hline & III-IV & 64(38.1) & 21 & 43 & \\
\hline \multirow[t]{2}{*}{ Microvascular invasion } & Absent & 95(56.55) & 52 & 43 & 0.029 \\
\hline & Present & 73(43.45) & 27 & 46 & \\
\hline \multirow[t]{2}{*}{ Child classification } & A & 158(94.05) & 75 & 83 & 0.751 \\
\hline & $B+C$ & $10(5.95)$ & 4 & 6 & \\
\hline \multirow[t]{2}{*}{ BCLC stage } & $0+\mathrm{A}$ & $83(49.4)$ & 47 & 36 & 0.020 \\
\hline & $\mathrm{B}+\mathrm{C}$ & $85(50.6)$ & 32 & 53 & \\
\hline \multirow[t]{2}{*}{ TMN stage } & I+II & $126(75)$ & 64 & 62 & 0.109 \\
\hline & III+IV & $42(25)$ & 15 & 27 & \\
\hline
\end{tabular}


Decision curve analysis (DCA) is a novel method for evaluating prediction with clinical net benefits. A wider range of threshold probability indicates better estimation of decision outcomes. As show in Figure 4, when compared with BCLC and TNM staging systems, GCLC-based nomograms for OS presented better net benefit, which indicated improved predictive value for the probabilities of OS at 3 and 5 years (Fig. 4G and 4H). Similarly, improved performance for predicting 3- and 5- year DFS was also presented according to GCLC-based nomogram for DFS (Fig. 4I and 4J).

A. HR for 5-year OS according to GCLC

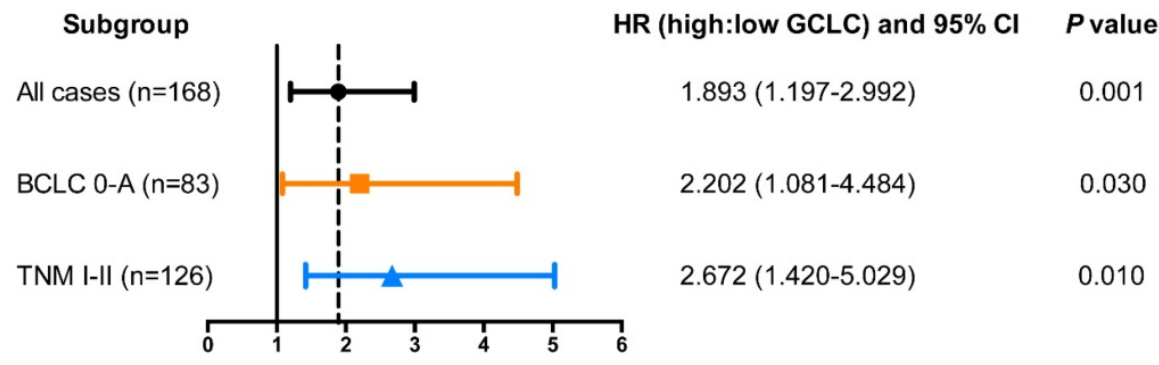

B. HR for 5-year DFS according to GCLC

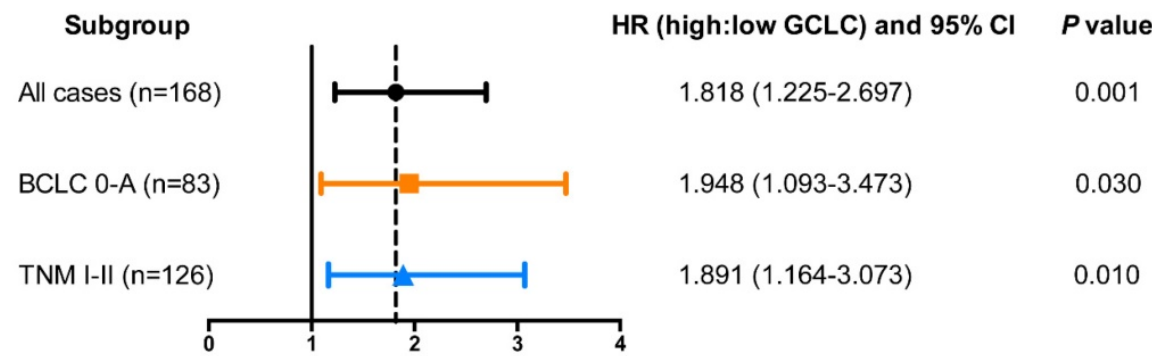

Figure 3. Tumor GCLC level is associated with $\mathbf{5}$-year OS and DFS in patients with early-HCC. Hazard ratios (HR) of tumor GCLC level for overall survival and disease-free survival in different subgroups of HCC patients. The HRs of tumor GCLC level for (A) 5-year OS and (B) DFS are obtained by comparing the patients with high tumor GCLC level with those with low tumor GCLC level. HR>1.0 is considered as a poorer outcome. BCLC, Barcelona Clinic Liver Cancer staging system. TNM, Tumor Node Metastasis staging system of AJCC 7th edition.

Table 2. Univariate and multivariate analysis of clinicopathological factors with OS and DFS in HCC patients.

\begin{tabular}{|c|c|c|c|c|c|c|}
\hline & \multicolumn{3}{|l|}{ OS } & \multicolumn{3}{|l|}{ DFS } \\
\hline & Univariate & Multivariate & & Univariate & Multivariate & \\
\hline Variables & $p$ value & HR(95\% CI) & $p$ value & $p$ value & HR( $(95 \% \mathrm{CI})$ & $p$ value \\
\hline Age, years (>50 vs. $\leq 50$ ) & 0.093 & & NS & 0.824 & & NS \\
\hline Gender (male vs. female) & 0.751 & & NS & 0.113 & & NS \\
\hline HBsAg (positive vs. negative) & 0.201 & & NS & 0.388 & & NS \\
\hline AFP, ng/ml (>20 vs. $\leq 20)$ & 0.003 & 2.028(1.233-3.335) & 0.005 & 0.002 & $1.695(1.115-2.578)$ & 0.014 \\
\hline CEA, ng/ml (>5 vs. $\leq 5)$ & 0.28 & & NS & 0.344 & & NS \\
\hline CA19-9, U/ml (>36 vs. $\leq 36)$ & 0.047 & & NA & 0.006 & $1.603(1.037-2.477)$ & 0.034 \\
\hline ALT, U/L (>40 vs. $\leq 40)$ & 0.278 & & NS & 0.046 & & NA \\
\hline AST, U/L (>37 vs. $\leq 37$ ) & 0.003 & & NA & 0.007 & & NA \\
\hline Y-GT, U/L (>54 vs. $\leq 54)$ & 0.001 & 1.714(1.047-2.807) & 0.032 & $<0.001$ & $1.624(1.060-2.488)$ & 0.026 \\
\hline Ascites (present vs. absent) & 0.068 & & NS & 0.029 & $2.155(1.060-4.381)$ & 0.034 \\
\hline Liver cirrhosis (yes vs. no) & 0.937 & & NS & 0.878 & & NS \\
\hline Tumor number (multiple vs. single) & 0.946 & & NS & 0.071 & & NS \\
\hline Tumor size, $\mathrm{cm}(>5$ vs. $\leq 5)$ & $<0.001$ & 2.271(1.444-3.573) & $<0.000$ & $<0.001$ & 2.348(1.576-3.499) & $<0.001$ \\
\hline Tumor encapsulation (complete vs. none) & 0.058 & & NS & 0.077 & & NS \\
\hline Tumor differentiation (III-IV vs. I-II) & 0.024 & & NA & 0.06 & & NS \\
\hline Microvascular invasion (present vs. absent) & 0.03 & & NA & 0.108 & & NS \\
\hline Tumor GCLC level (high vs. low) & 0.002 & 1.893(1.197-2.992) & 0.006 & 0.003 & $1.818(1.225-2.697)$ & 0.003 \\
\hline
\end{tabular}

Abbreviations: OS, overall survival; DFS disease-free survival; HBsAg, hepatitis B surface antigen; AFP: afetoprotein; CEA: carcinoembryonic antigen; CA19-9: carbohydrate antigen 19-9; ALT: alanine aminotransferase; AST: aspartate aminotransferase; $\gamma$-GT, $\gamma$-glutamyl transferase; HR, hazard ratio; CI, confidential interval; NA, not adopted; NS, not significant. Data obtained from the Cox proportional hazards model, $P<0.05$ was considered as statistically significant. 
A.

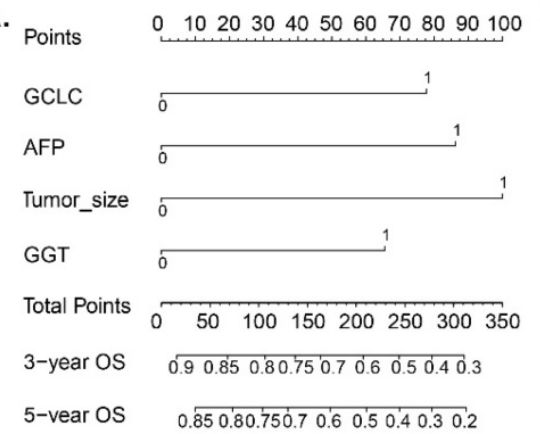

B.

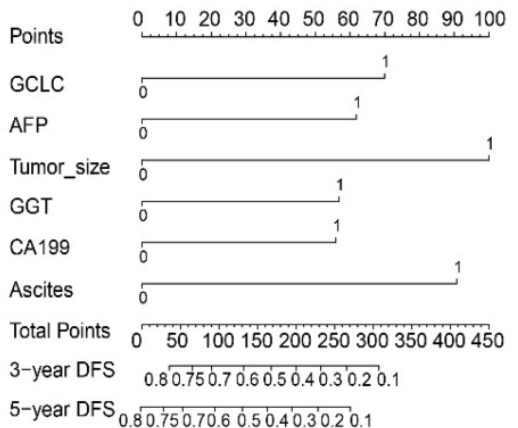

C.

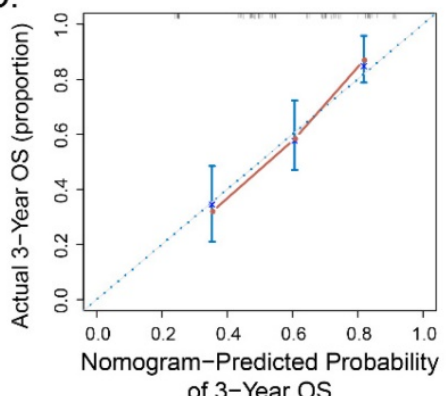

E.

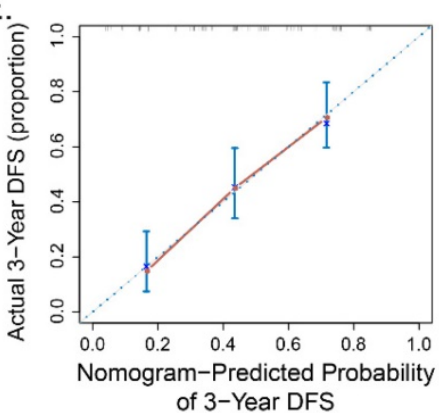

D.

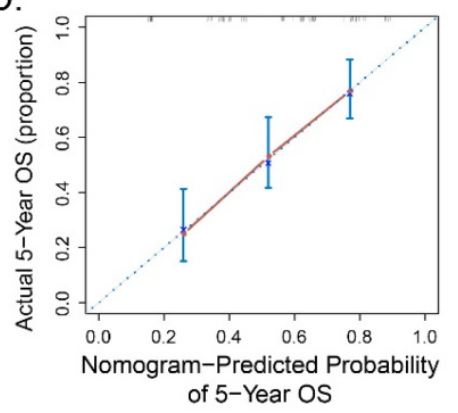

F.

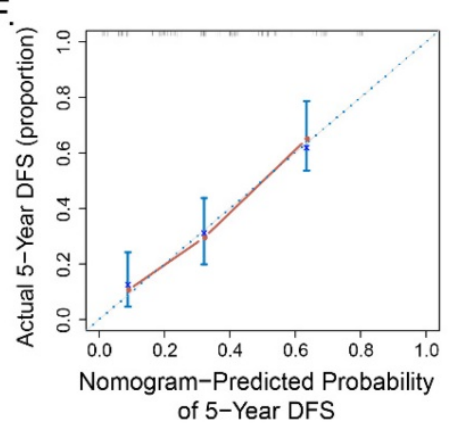

G. Assessment of 3-year OS with DCA

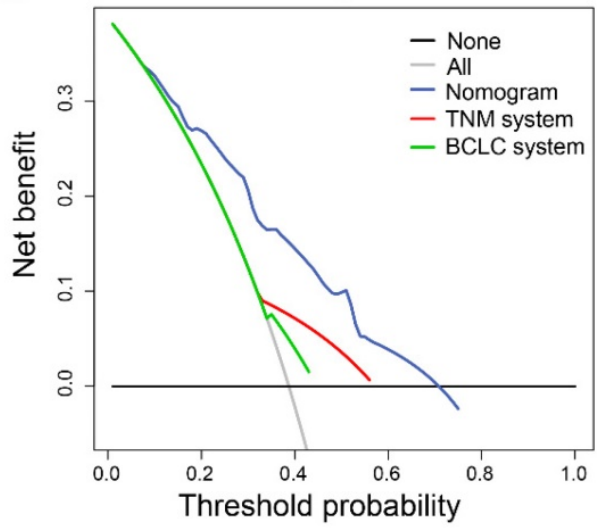

I.

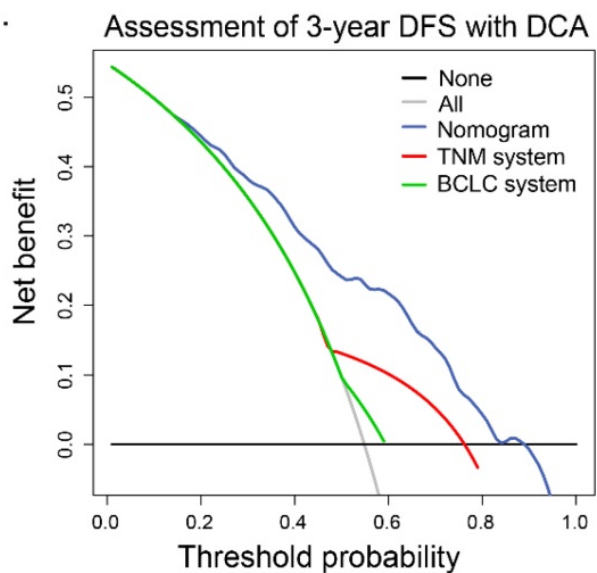

H. Assessment of 5-year OS with DCA

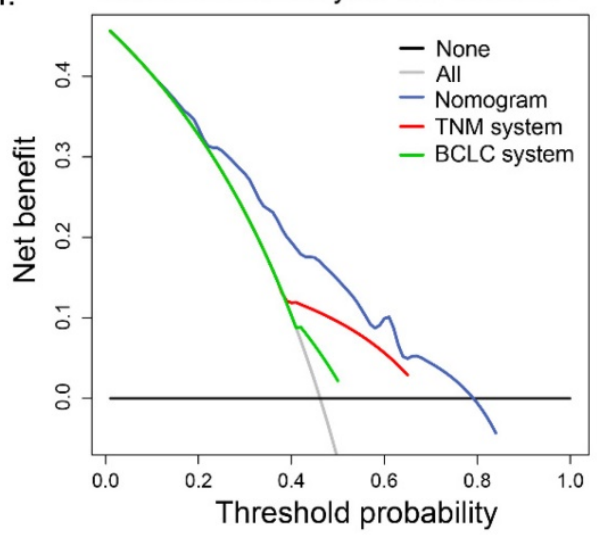

J.

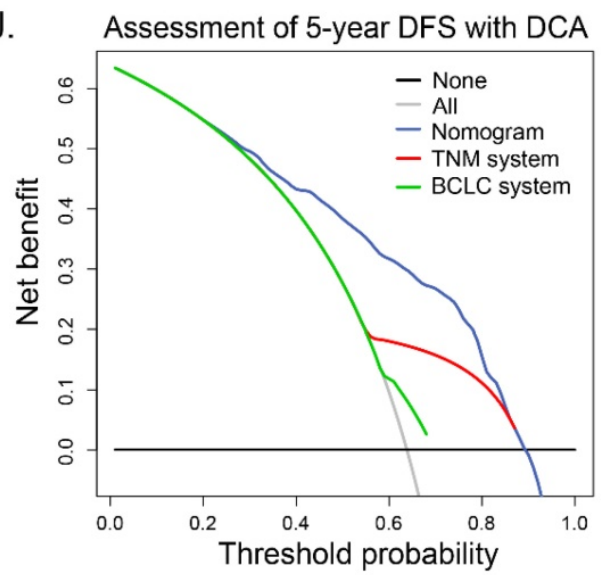

Figure 4. Prognostic nomograms and calibration curves and decision curve analysis. Nomogram predicted OS (A) and DFS (D) for HCC patients. To get the probability of OS or DFS, a vertical line from the factors to the points scale first was draw and a point for each factor then was obtain. The points of all factors was summed up to get a total points. Finally, a vertical line from the total point scale to the probability scale was drawn and the probability of OS or DFS was gotten. Calibration curves for 3-and 5- year OS (B, C) and 3- and 5- year DFS (E, F) was shown. Probability of OS or DFS predicted by nomograms is plotted on the X axis and the observed OS or DFS is plotted on the $Y$ axis. Decision curve analyses show the clinical benefit among different models. Nomogram-predicted probabilities of 3-year OS and DFS (G, H), 5-year OS and DFS (I, J) are compared with routine staging system. Dashed lines: clinical net benefits across a range of threshold probabilities; the horizontal solid black line: to assume no patients will experience the event; the solid gray line: to assume all patients will experience the event. BCLC, Barcelona Clinic Liver Cancer staging system. TNM, Tumor Node Metastasis staging system of AJCC $7^{\text {th }}$ edition. 
Table 3. Discriminatory capabilities of nomogram and independent prognostic factors in patients with HCC: C-indices in OS and DFS prediction.

\begin{tabular}{|c|c|c|c|c|}
\hline \multirow[t]{2}{*}{ Variables } & \multicolumn{2}{|l|}{ Overall survival } & \multicolumn{2}{|c|}{ Disease-free survival } \\
\hline & C-index $(95 \% \mathrm{CI})$ & $P$ value & C-index $(95 \% \mathrm{CI})$ & $P$ value \\
\hline GCLC & $0.591(0.538-0.644)$ & & $0.577(0.530-0.624)$ & \\
\hline TNM & $0.582(0.533-0.631)$ & & $0.594(0.553-0.635)$ & \\
\hline TNM + GCLC & $0.637(0.577-0.697)$ & $0.071 \dagger$ & $0.636(0.583-0.689)$ & $0.220 \dagger$ \\
\hline BCLC & $0.542(0.487-0.597)$ & & $0.545(0.497-0.593)$ & \\
\hline BCLC + GCLC & $0.604(0.544-0.664)$ & $0.009+$ & $0.594(0.539-0.649)$ & $0.021 \dagger$ \\
\hline Nomogram & $0.701(0.645-0.757)$ & & $0.707(0.658-0.756)$ & \\
\hline Nomogram vs. TNM & & $<0.001 \ddagger$ & & $<0.001 \ddagger$ \\
\hline Nomogram vs. BCLC & & $<0.001 \ddagger$ & & $<0.001 \ddagger$ \\
\hline \multicolumn{5}{|c|}{$\begin{array}{l}\text { Abbreviations: OS, overall survival; DFS, disease-free survival; C-index, } \\
\text { concordance index; CI, confidence interval; TNM, Tumor-Nodes-Metastases; } \\
\text { BCLC, Barcelona Clinic Liver Cancer. } \\
\text { t: Compared the C-index with the original model with or without GCLC } \\
\text { expression data; ł: Compared the C-index of nomogram with TNM stage and } \\
\text { BCLC stage in patients with HCC. }\end{array}$} \\
\hline
\end{tabular}

\section{Discussion}

In the present study, this is the first report on the relationship between tumor GCLC level and the postoperative clinical outcome of HCC patients. We enrolled a large cohort containing 168 patients with HCC who received curative resection, and demonstrate that GCLC level is higher in tumor tissues than in peritumoral tissues. In accordance with these finding, GCLC is highly transcriptionally activated in tumor. We also show that tumor GCLC correlates to tumor differentiation, microvascular invasion and BCLC stage. Moreover, univariate and multivariate analyses was able to demonstrate that tumor GCLC acts as an independent prognostic factor for OS and DFS. Patients with high tumor GCLC level have poorer OS and DFS than those with low tumor GCLC level. Importantly, our data indicated that tumor GCLC can still predict the outcome in patients with early-stage HCC for whom routine staging systems fail to give an accurate prediction. However, Mougiakakos et al. [22] reported that high GCLC expression predicted better prognosis in malignant melanoma, which seem to be inconsistent with our finding. We thought that gene polymorphism of GCLC might contribute to this discrepancy [23]. The gene polymorphism of GCLC was found to be closely related to GCL activity and different kinds of GCLC genotype have different association with outcomes of tumor. Therefore, further research remains to be conducted to explore the specific gene polymorphism of GCLC which correlates with prognosis of HCC patients.

Disrupted intracellular redox status has been frequently observed in cancer cells, owing to the impaired balance between ROS generation and elimination [6]. Increasing levels of ROS, which lead to a condition known as oxidative stress, can be toxic to the cancer cells. To adapt to such persistent stress, malignant cells constitutively overexpress antioxidant genes which involve in scavenging ROS. As consequence, cancer cells develop an enhanced antioxidant capacity and become resistant to both endogenous and exogenous stress [24]. Nuclear factor E2-related factor 2 (NRF2) is an important transcriptional factor that regulates the expression of a wide range of antioxidant genes by binding to the antioxidant-response element (ARE) in their promotors [25]. Several reports have shown that high level of NRF2 expression was related to poor prognosis in cancers [26-27]. Saito et al. [28] reported that NRF2-dependent metabolic reprogramming contributed to malignancy of HCV-related HCC, which suggests that the target genes of NRF2 may be responsible for the malignant phenotype of HCC. GCLC is one of the typical target genes regulated by NRF2 and high level of GCLC was found to correlate with the content of GSH, a key antioxidant [11]. GSH is proved to be associated with tumor proliferation, metastasis. These facts may partially explain why high tumor GCLC level is associated with poor outcome in HCC patients but its mechanism need to be further investigated.

Nomograms are widely used as prognostic models in oncology and medicine, with the ability to generate an individual probability of a clinical event by integrating diverse prognostic variables [29]. In the present study, for the first time, we established two nomograms that integrate tumor GCLC level, AFP, tumor size and GGT to improve the prognostic accuracy of tumor GCLC level in HCC patients. DCA was first used by Vickers and Elkin as a novel analytical device to determine whether a predictive model is useful or not [30]. By performing DCA, we demonstrate that GCLC-based nomograms present a better net benefit than routine staging systems including BCLC and TNM staging system. Therefore, GCLC-based nomograms can be a novel predictive model with high accuracy for HCC patients.

In conclusion, our results indicates that high tumor GCLC level is associated with the malignant phenotype of HCC and tumor GCLC level can predict the postoperative clinical outcome in patients, even those with early stage HCC. Moreover, GCLC-based nomogram can provide a more accurate prognostic assessment as compared to routine clinical staging systems. Thus, GCLC is a potential prognostic biomarker for HCC patients who receive curative resection. Targeting GCLC may be a helpful strategy to control tumor relapse and prolong survival after curative resection; they await further investigation.

\section{Supplementary Material}

Supplementary figure and table.

http://www.jcancer.org/v10p3333s1.pdf 


\section{Acknowledgements}

We thank Dr. Hui Sun from GenomiCare Biotechnology (Shanghai) Co. for statistical assistance.

This work was supported by grants from the China National Natural Science Foundation (81272733) and the "973" State Key Basic Research Program of China (2014CB542101).

\section{Competing Interests}

The authors have declared that no competing interest exists.

\section{References}

[1] Njei B, Rotman Y, Ditah I, Lim JK. Emerging trends in hepatocellular carcinoma incidence and mortality. Hepatology 2015; 61(1):191-9.

[2] Llovet JM, Burroughs A, Bruix J: Hepatocellular carcinoma. Lancet. 2003; 362:1907-17.

[3] Sherman M. Recurrence of hepatocellular carcinoma. N Engl J Med 2008; 359:2045-7.

[4] Wang JH, Changchien CS, Hu TH, et al. The efficacy of treatment schedules according to Barcelona Clinic Liver Cancer staging for hepatocellular carcinoma: survival analysis of 3,892 patients. Eur J Cancer. 2008; 44:1000-6.

[5] Assi M. The differential role of reactive oxygen species in early and late stages of cancer. Am J Physiol Regul Integr Comp Physiol. 2017; 313:646-653.

[6] Trachootham D, Alexandre J, Huang P. Targeting cancer cells by ROS-mediated mechanisms: a radical therapeutic approach? Nat Rev Drug Discov. 2009; 8:579-91.

[7] Terradez P, Asensi M, Lasso de la Vega MC, et al. Depletion of tumour glutathione in vivo by buthionine sulphoximine: modulation by the rate of cellular proliferation and inhibition of cancer growth. Biochem J. 1993 Jun; 292:477-83.

[8] Huang Z, Chen C, Zeng Z, el al. Mechanism and significance of increased glutathione level in human hepatocellular carcinoma and liver regeneration. FASEB J. 2001 Jan; 15:19-21.

[9] Carretero J, Obrador E, Anasagasti MJ, et al. Growth-associated changes in glutathione content correlate with liver metastatic activity of B16 melanoma cells. Clin Exp Metastasis. 1999; 17:567-74.

[10] Mena S, Benlloch M, Ortega A, et al. Bcl-2 and glutathione depletion sensitizes B16 melanoma to combination therapy and eliminates metastatic disease. Clin Cancer Res. 2007 May; 13:2658-66.

[11] Traverso N, Ricciarelli R, Nitti $M$, et al. Role of glutathione in cancer progression and chemoresistance. Oxid Med Cell Longev. 2013; 2013:972913.

[12] Järvinen K, Soini Y, Kahlos K, Kinnula VL. Overexpression of gamma-glutamylcysteine synthetase in human malignant mesothelioma. Hum Pathol. 2002 Jul; 33:748-55.

[13] Kim AD, Zhang R, Han X, et al. Involvement of glutathione and glutathione metabolizing enzymes in human colorectal cancer cell lines and tissues. Mol Med Rep. 2015 Sep; 12:4314-4319

[14] Li M, Zhang Z, Yuan J, Zhang Y, Jin X. Altered glutamate cysteine ligase expression and activity in renal cell carcinoma. Biomed Rep. 2014 Nov; $2 \cdot 831-834$

[15] Nguyen A, Loo JM, Mital R, et al. PKLR promotes colorectal cancer liver colonization through induction of glutathione synthesis. J Clin Invest. 2016 Feb; 126:681-94.

[16] Fiorillo M, Sotgia F, Sisci D, et al. Mitochondrial "power" drives tamoxifen resistance: NQO1 and GCLC are new therapeutic targets in breast cancer. Oncotarget. 2017 Mar ; 8:20309-20327.

[17] Hiyama N, Ando T, Maemura K, et al. Glutamate-cysteine ligase catalytic subunit is associated with cisplatin resistance in lung adenocarcinoma. Jpn J Clin Oncol. 2018 Apr; 48:303-307.

[18] Lin L, Chen C, Ho C, et al. $\gamma$-Glutamylcysteine synthetase ( $\gamma$-GCS) as a target for overcoming chemo- and radio-resistance of human hepatocellular carcinoma cells. Life Sci. 2018 Apr; 198:25-31.

[19] Liu CW, Hua KT, Li KC et al. Histone Methyltransferase G9a Drives Chemotherapy Resistance by Regulating the Glutamate-Cysteine Ligase Catalytic Subunit in Head and Neck Squamous Cell Carcinoma. Mol Cancer Ther. 2017 Jul; 16:1421-1434.

[20] Qian Y, Zhang J, Wu W, et al. P48 is a predictive marker for outcome of postoperative interferon-alpha treatment in patients with hepatitis B virus infection-related hepatocellular carcinoma. Cancer. 2006 Oct; 107:1562-9.

[21] Altman DG, McShane LM, Sauerbrei W, Taube SE. Reporting Recommendations for Tumor Marker Prognostic Studies (REMARK): explanation and elaboration. PLoS Med. 2012 May; 9(5):e1001216.

[22] Mougiakakos D, Okita R, Ando T, et al. High expression of GCLC is associated with malignant melanoma of low oxidative phenotype and predicts a better prognosis. J Mol Med (Berl). 2012 Aug; 90:935-44.
[23] Nichenametla SN, Muscat JE, Liao JG, et al. A functional trinucleotide repeat polymorphism in the 5 -untranslated region of the glutathione biosynthetic gene GCLC is associated with increased risk for lung and aerodigestive tract cancers. Mol Carcinog. 2013 Oct; 52:791-9.

[24] Pervaiz S, Clement MV. Tumor intracellular redox status and drug resistance--serendipity or a causal relationship? Curr Pharm Des. 2004; 10:1969-77.

[25] Uruno A, Motohashi H. The Keap1-Nrf2 system as an in vivo sensor for electrophiles. Nitric Oxide. 2011 Aug; 25:153-60.

[26] Zhang M, Zhang C, Zhang L, et al. Nrf2 is a potential prognostic marker and promotes proliferation and invasion in human hepatocellular carcinoma. BMC Cancer. 2015 Jul 21;15:531.

[27] Lu K, Alcivar AL, Ma J, et al. NRF2 Induction Supporting Breast Cancer Cell Survival Is Enabled by Oxidative Stress-Induced DPP3-KEAP1 Interaction. Cancer Res. 2017 Jun; 77:2881-2892.

[28] Saito T, Ichimura Y, Taguchi K, et al. p62/Sqstm1 promotes malignancy of $\mathrm{HCV}$-positive hepatocellular carcinoma through Nrf2-dependent metabolic reprogramming. Nat Commun. 2016 Jun; 7:12030.

[29] Balachandran VP, Gonen M, Smith JJ, DeMatteo RP. Nomograms in oncology: more than meets the eye. Lancet Oncol. 2015; 16:e173-80.

[30] Vickers AJ, Elkin EB. Decision curve analysis: a novel method for evaluating prediction models. Med Decis Making. 2006 Nov-Dec; 26:565-74. 